\title{
Spatial Interaction between Lifestyles and Tuberculosis: An Expert and Public Participatory GIS in Malaysia
}

\author{
Ridzuan, N. A., ${ }^{1}$ Abdul Rasam, A.R., ${ }^{1 *}$ Isa, M. M. ${ }^{2}$ and Shafie, F. A. ${ }^{3}$ \\ ${ }^{1}$ Centre of Studies for Surveying Science and Geomatics, Faculty of Architecture, Planning and Surveying, \\ Universiti Teknologi MARA, Shah Alam, Selangor, Malaysia E-mail: rauf@uitm.edu.my \\ ${ }^{2}$ Centre of Studies for Landscape Architecture, Faculty of Architecture, Planning and Surveying, Universiti \\ Teknologi MARA, Shah Alam, Selangor, Malaysia \\ ${ }^{3}$ Centre of Environmental Health and Safety, Faculty of Health Sciences, Universiti Teknologi MARA, \\ Puncak Alam, Selangor, Malaysia \\ *Corresponding Author
}

DOI: https://doi.org/10.52939/ijg.v17i5.2033

\begin{abstract}
Human lifestyles are potentially contributing factors to tuberculosis (TB) transmission in Malaysia, but previous studies have not comprehensively explored these factors from geospatial and public perspectives. The aim of the study was to examine the impacts of lifestyle risk factors on TB cases in Shah Alam using selected experts and public participatory GIS (PPGIS) approach with the 5-risk scale from 1 to 5. Two local health experts have suggested that the overall risk scale of TB-lifestyle factors are 2.33 (medium risk) as revealed in the public perception and GIS map (2.08). Key findings have shown the overall factor has a minimal correlation with TB, but the individual factor of obesity, diet routine, living conditions, physical exercise and socioeconomic status become a potential threat to the local community to contract TB infection, especially if all these factors are combined together.
\end{abstract}

\section{Introduction}

Lifestyle refers to a way of living that is practised by individuals, groups and nations and is formed by specific geographical, economic, political, cultural and religious aspects. Lifestyle can be also defined as the characteristics of the inhabitants of a region in an extraordinary time and place. It includes day to day behaviours and functions of individuals in job, activities, fun and diet (Farhud, 2015). In recent decades, researchers have become more interested in discussing lifestyle as a significant factor in health. According to the World Health Organization (WHO), $60 \%$ of related factors to individual health and quality of life are correlated with lifestyle. Since millions of people live an unhealthy lifestyle, they are more likely to suffer from illness, disability and even death. Thus, the relationship between lifestyle and health should be highly considered. Based on the findings of-existing studies, it can be said that lifestyle has significant influences on physical and mental health of human beings, but there are different forms of such influences (Farhud, 2015).

Tuberculosis (TB) is one of the airborne diseases. TB is well known to be associated with poverty and multiple social factors (Narasimhan et al., 2013, Toque et al., 2001 and Rasam et al., 2018). However, relatively little attention has been paid to the behavioural and lifestyle factors, apart from those associated with drug abuse, alcoholism and HIV infection (Chang and Leung, 2008 and Mohammed et al., 2011). In recent years, the roles of other lifestyle related factors have been increasingly recognised. In particular, smoking has been associated with excess risks of TB infection, disease and mortality (Chang and Leung, 2008). Roughly 9.2 million new TB cases and 1.7 million TB-related deaths were reported in 2006 (Ibrahim et al., 2011).

TB is most commonly spread among family members, close friends, and people who live in our immediate vicinity. TB is caused by a microorganism called Mycobacterium which could be an infection or irresistible infection. It is spread from one person to another. A few strains of Mycobacterium tuberculosis have created medicare resistance, which can make treatment of the infection troublesome. In case untreated, TB can cause serious impacts on wellbeing. A person's health is routinely compromised by inhaling germs. These germs are released into the air when a person suffering from an active disease coughs. In addition, the related microscopic organisms Mycobacterium bovis and Mycobacterium africanum can moreover 
cause the TB. The body's reaction to dynamic TB contamination produces irritation that can harm the lungs. Zones influenced by dynamic TB steadily fill with scar tissue. Side effects of dynamic TB of corporate hacking that keeps going for three or more weeks, chest torments or trouble taking full breaths, inadvertent weight misfortune, depletion or weariness, hacking up blood, fever and chills, night sweats and the misfortune of craving. The lifestyle of an individual also can contribute to the development of TB.

Poverty or low socioeconomic status, poor living conditions, or overcrowding is just a few of the many factors that commonly influence TB. (Andrejs et al., 2016, Cramm et al 2011, Kashyap et al., 2016, Lopez De Fede et al., 2008, Muniyandi et al., 2011, Olson et al., 2012 and Yen et al., 2017), malnutrition and other related disease infection (Leung et al., 2007, Lin et al., 2018, Lonnroth al., 2010 and Mohammed et al., 2011).

The case-control study in Erbil city has shown that around one quarter of the cases were smokers compared among other controls (Ibrahim et al., 2011). Based on the previous studies, the highest percentages of TB disease were smokers compared to other factors (Change and Leung, 2008). According to the Department of Health's Central Health Education Unit, Hong Kong (Ngai, 2002), the best weapon is our own defence system by paying attention to personal hygiene, adopting a healthy lifestyle and eating a well-balanced diet, getting adequate rest and exercise, not smoking or drinking alcohol, keeping the environment clean and avoiding poorly ventilated areas. However, risk factors associated with TB can change over time. In Malaysia, there are several local attempts to link environmental factors and TB using GIS technology (Abdul Jalil and Abdul Rasam, 2021, Abdul Rasam et al., 2016 and 2017 and Rosli et al., 2018, Abdul Rasam et al., 2020a and Rajab et al., 2020), but this study did not examine in depth the lifestyle factors with TB pattern.

Besides this, most of the previous studies only applied cross-sectional or case-control studies, to control all the potential confounders adequately (Leung et al., 2007, Lienhard et al., 2003 and Lin et al., 2013). Only a few studies relate this statistical method with advanced GIS techniques. For example, in a prospective study conducted in Hong Kong, the annual TB notification rates were 735 , 427, and 174 per 100000 current smokers, exsmokers and never-smokers respectively (Change and Leung, 2008). Thus, the purpose of this study is to apply spatial analysis and GIS mapping to examine the effects of lifestyle risk factors for TB cases and to explore the capability of GIS to study the lifestyle factors and airborne disease distribution. The objectives of the study are:

i. To identify the lifestyle risk factors contributing to TB

ii. To produce a risk factor map of lifestyle risk effect on TB

iii. To determine the correlation between lifestyle factors and TB

Combination of GIS, statistical and publicquestionnaire surveys can explore how much lifestyle risk factors contribute to TB occurrences. Chang (2019) stated a GIS is a computer system for gathering, managing, analysing, and presenting geographical data. It has been increasingly applied in many disciplines such as spatial epidemiology, in particular the association between pathological factors (e.g. TB) and their spatial environments (Cromley, 2003, Davis et al., 2018, Rasam et al., 2019, Rasam et al., 2018 and Abdul Rasam et al., 2020b) disease occurrences.

\section{Literature Review}

\subsection{Airborne Disease and Tuberculosis}

Airborne disease is a disease that is related to breathing. The term "airborne infection" refers to any disease that is caused by pathogens that are spread through the air. The pertinent pathogens seem to moreover be infections, microscopic organisms, or organisms. The pathogens transmitted could also be any reasonably microorganism, and that they could also be unfolded in aerosols, dirt or liquids. Airborne disease can spread by hacks, sniffles, talks, retching nasal and throat discharges into the air. The viruses or bacteria that out of a person who coughs, or sneezes cannot be seen clearly. Airborne pathogens or allergens frequently cause irritation within the nose, throat, sinuses and the lungs. This is often caused by the inward breath of these pathogens that influence a person's respiratory system or the rest of the body. Sinus clog, hacking and sore throats are cases of irritation of the upper respiratory discuss way due to these airborne agents. This situation plays a noteworthy part in airborne infections, which is connected to asthma. Toxins are said to impact lung work by expanding discussion of irritation. Because of that, the airborne disease is hard to control because it travels in the air.

Airborne disease is related to air pollution. In 2016, there were an evaluated 1.3 million TB deaths among HIV-negative individuals which have decreased from 1.7 million in 2000. But, an extra 374000 deaths among HIV-positive individuals occurred. 
An evaluated the World Health Organization (WHO) almost 10.4 million individuals fell sick with TB in 2016 is $90 \%$ were adults, $65 \%$ were male, $10 \%$ were individuals living with HIV, which is $74 \%$ in Africa and $56 \%$ were in five nations which are India, Indonesia, China, the Philippines and Pakistan.

TB is one of types of airborne disease that could be an illness caused by the bacteria Mycobacterium. It is an irresistible illness that can harm a person's lungs, but can cause harm to other parts of the body (CDC, 2011). Genetic studies that were reviewed by Michael Iseman (2013) show that tuberculosis has been present for at least 15,000 years. TB has been around for tens of thousands a long time. TB may be a ponder wellbeing issue in numerous nations, particularly in creating a nation. It has been called a "disease of poverty" since it is more predominant in places with challenging financial conditions that lead to a swarmed living, lack of healthy sustenance, and numerous more.

\subsection{Lifestyle Factors on Tuberculosis}

Each disease develops with many reasons due to many aspects such as social behaviours and lifestyle factors. However, the burden of any disease cannot be defined without first examining how it affects the person and also their daily habits and their lifestyle. There are many lifestyle factors that can cause TB diseases.

\subsubsection{Smoking}

Smoking is inward breath, chewing, or sniffing tobacco or tobacco- related products (Ibrahim et al., 2011 and Brunet et al., 2011). Smoking is the key risk factor for a few dangerous infections like cancer, heart infection, stroke and tuberculosis (ERS, 2021). The bacteria that cause TB are almost around one-third of the world's population. Smoking is an unfortunate action. Smoking or introduction of smoke is one of the risks for development of TB. The relationship between smoking and TB has been examined as early as 1918. Roughly 8.8 million individuals in 2003 had active tuberculosis and records for almost 1.7 million deaths around the world each year. Report from JAMA and Archives Journals (2007) stated that concurring the ponders, individuals who smoked had a 73 percent increase in the unexpected of getting tainted with $\mathrm{TB}$ and were more than twice as likely to form dynamic TB as those who did not smoke. Smoking might decrease safe reaction or harm the work of cilia within the aviation routes, expanding the chance for TB. Smoking decreases the life of a male by approximately 12 a long time and the life of a female by around 11 a long time.
Worldwide, around 1 billion grown-ups, which are 800 million men and 200 million ladies, directly smoke cigarettes. This can be an underestimate of adding up to tobacco exposure around the world, because it does not incorporate childhood smoking, smokeless tobacco or second-hand smoke. Cigarette smoking predominance changes broadly around the world. Over $80 \%$ of the world's grown-up male smokers, while and half of the world's female smokers who live in moo or middle-income nations. In the interim, tobacco slaughters nearly 6 million individuals around the world each year, with about $80 \%$ of these deaths. Each year 600,000 nonsmokers around the world pass on from the introduction of natural tobacco smoke. By 2030 tobacco will slaughter an anticipated 8 million individuals around the world each year. Tobacco use caused 100 million deaths worldwide amid the 20th century, and if current patterns proceed it'll slaughter 1 billion individuals within the $21 \mathrm{st}$ century.

\subsubsection{Alcohol drinking}

Alcohol drinking is associated with the use of alcoholic beverages such as ethyl alcohol or ethanol. It is regarded as typical social behaviour. (Suhadev et al., 2011). Alcohol and any substance abuse are also risk factors for TB. There are numerous dangers related to all forms of excessive drinking, especially alcohol use disorder (AUD). Heavy drinking and orgy drinking can put people at risk of numerous serious issues such as ranging from car crashes while drunk to liver failure or cancer. One risk related to excessive drinking is tuberculosis. BMC Public Health confirmed that tuberculosis has been associated with alcohol misuse.

The World Health Organization (WHO) reviewed that alcohol abuse can increase the danger of contracting TB. People who abuse alcohol are much less likely to eat regular, healthy meals and malnutrition adds extra risk to those who may additionally contract TB. The development of TB can increase by the use of harmful alcohol. TB has become fantastically concentrated in responsive groups in intermediate and low-incidence nations. Harmful alcohol use can be an imperative populace level hazard issue for TB and an excessive incidence of alcohol use disorders. A complete care package deal is important to identify trouble drinkers, diagnose alcohol use disorder, and refer to splendid alcohol interventions. A few countries have analysed efficient screening for harmful alcohol use of all TB patients. Screening and diagnosis of other mental health problems may also be sanctioned. 


\subsubsection{Physical exercise}

Lack of physical exercise is one of the lifestyle factors that can affect chronic diseases (Booth et al., 2012) that can indirectly increase the infection of TB diseases. For TB patients, however, physical activity can be beneficial. Exercise can help our body fight the TB infection, trigger recovery and improve our mood. For individuals who are currently affected with tuberculosis they can perform a simple activity like brisk walking outside. It can boost to provide the body with fresh air and workout to assist adaptation of the bacteria. Physical exercise activities in this study were defined as practicing sports or exercise such as walking, jogging, and swimming for 30 minutes or more in a day.

\subsubsection{Nutritional status}

According to the World Health Organization (WHO), malnutrition is attributed to insufficiency, excesses or inequality in a person's intake of energy or nutrients. The terminology malnutrition can be divided into two groups of conditions. One is under nutrition which incorporates hindering, which is moo tallness for age, squandering which is moo weight for tallness, underweight, such as moo weight for age and micronutrient lacks or insufficiencies comparatively an insufficiency of vital vitamins and minerals. The other one is overweight, corpulence and diet-related noncommunicable illnesses such as heart illness, stroke, diabetes and cancer. WHO (2018) stated the malnutrition associated with poverty or poor diet puts humans at risk of TB.

Malnutrition can speed up the risk of TB and at the same time TB can lead to malnutrition. Human beings with TB are extremely prone to malnutrition. While appropriate TB cure can help normalize nutritional status. Besides, many TB sufferers are still malnourished at the end of TB treatment. Malnutrition with poverty and poor diet can increase the risk of TB. Under nutrition is among the most vital decisions of TB. For TB interception, by remodelling nutritional fame at populace level is vital. This has to be part of broader movements on social populace. Evidence shows that a decrease in body mass index (BMI) is associated with an increased risk of TB. Micronutrient deficiencies such as a low plasma concentration of vitamin A, Zinc, Iron and vitamin D also correlated with the risk of tuberculosis.

\subsubsection{Obesity}

Obesity is one of the risk factors of TB. Obesity can reach anyone at any time, regardless of gender or age. According to the World Health Organization, the challenges of the 21 st century are childhood obesity. It is one of the most deliberate global public healthiest that affects every country in the world. Leung (2007) concludes that obesity is associated with a lesser risk of active pulmonary tuberculosis in the older population of Hong Kong. In 2016, 216 million were classified as overweight but not obese in 2016. This obese condition also affects younger children over 38 million children aged under 5 living with overweight or obese in 2017. Overweight and weight issues are major risk components for an incessant sickness, counting of diabetes, and diabetes one of the major dangers of TB. Individuals with low BMI have a higher TB morbidity (Lonnroth et al., 2010).

\subsubsection{Socio-economic status (SES)}

Chronic illnesses such as tuberculosis cause impressive dreariness and mortality. The socioeconomic status is also one of the factors that can cause TB diseases. Tuberculosis (TB) influences the foremost productive age group and the resultant financial cost to society is high. Levels of destitution have ordinarily had a relationship to the burden of any illness. A few things have appeared that the financial status may be a more noteworthy risk factor for improvement of dynamic TB. Within the past, pondering a month to month salary was not related to active TB, but in Ethiopia the forecast of salary based on compensations do not reflect the real salary of people (Lope et al., 2008). In any case, destitute lodging conditions which could be an agent the financial status was related to dynamic TB.

Previous reports also proved that a poor household and crowding were the dominant risk factors for the development of TB. A past casecontrol study illustrated that those who impacted tuberculosis had been competent in a concentration of social unsettling influences, such as residential conflict, private and word related changes, and individual emergencies amid the two a long time before the improvement of tuberculosis. For the household person level, the predominance of TB is for the most part seen in middle-income nations South Africa, Zambia and Bangladesh are known to have a prevalence of at least 6 times higher than any other nation according to the World Health Organization. Socioeconomic status also can increase the risk for TB.

\subsubsection{Living conditions}

Living conditions such as crowded areas or busy areas can contribute to the TB infection (Baker et al., 2008). This is because the air pollution level is very high and can cause TB diseases related to 
breathing. Overcrowding creates a major health challenge and subsidizes the spread of TB infection among people who live in densely populated urban areas. Meanwhile, people who live in poverty have high chances to be infected by TB diseases. Poverty also contributed to an increasing number of individuals threatened by TB. Individuals who remained in 3-bedroom houses demonstrated that such a stuffed way of life and behaviour displayed a noteworthy challenge to their wellbeing, and the same similarity connected to individuals who remained in casual shacks.

Based on the study by Mmakala Esther in Ehlanzeni district, it was found that unemployment and unfortunate living conditions contributed to the predominance of TB in different communities of the locale. Since unemployment may be a major cause of destitution and leads to lack of healthy sustenance, destitution inclines people to TB. In this way, viable TB anticipation and control is conceivable inside a made strides socio-economic system.

\subsubsection{HIV}

The Human immunodeficiency virus is well known as HIV. HIV is a virus. Some viruses, such as the ones that cause colds or flu, stay in the body for only a few days. HIV, however, never goes away. HIV cannot be treated as other diseases. According to the Centers for Disease Control and Prevention, USA (2015) TB is one of the leading causes of death among people living with HIV. Individuals who are living with HIV are 26-31 times more likely to be vitiated with TB microscopic organisms. As of 2011, CDC anticipated $6 \%$ of all TB cases and $10 \%$ of TB cases among individuals matured 25-44 happened between individuals who were HIVpositive. The CDC suggests that all HIV positive individuals get reliable TB. HIV co-infection isn't the hazard calculated among TB contamination, but there are numerous other chance variables within the transmission of the illness.

\subsubsection{Diabetes}

Diabetes triples the threat of TB (Jeon and Murray, 2008). Consequently, rates of TB are higher in people with diabetes than in the general population, and diabetes is common co morbidity in people with TB. Diabetes can aggravate the medical path of TB, and TB can aggravate glycemic control in human beings with diabetes. Individuals with each condition for that reason require careful scientific management. Techniques are wished to create beyond any doubt that most effective care is given to sufferers with each infection. Diabetes occurrence is expanding universally. The foremost sensational broadening is in moo- and centre benefits countries display to handle rapid financial, social, and lifestyle changes. Advance increment within the number of diabetes-associated TB instances risks jeopardizing advancement that has been made within the worldwide battle in restriction to TB. In this manner, avoidance and care of diabetes must be a need not exclusively for partners concerned with care and overuse of non-communicable maladies, but moreover for those working on TB care and anticipation.

\subsection{GIS and Public Participation in Human Health and Lifestyles}

Within the 1960s and 1970s, the beginning of Geographical Information System (GIS) in its present frame lies within the application of quickly creating computing instruments, pre-eminently computer design in an assortment of areas such as urban planning, land administration and geocoding. Roger Tomlinson developed the first operational GIS for storing, manipulating and analysing data collected for Canada Land Inventory in 1984. GIS enables quick and easy access to large volume data to the user. A GIS is a computer system that provides capturing, storing, querying, analysing and displaying geospatial data (Chang, 2019).

The exceptional conceivable of GIS to advantage the wellbeing care industry is presently commencing to be realized. Both open and private divisions are making dynamic ways to saddle the information integration and spatial visualization quality of GIS. The sorts of companies and organizations embracing GIS span the wellbeing care range from open wellbeing divisions and open wellbeing arrangements and research agencies to hospitals, medical centres, and wellbeing protection organizations. The core geospatial technology of GIS is related to a number of fields, including remote sensing, Global Positioning System (GPS), Cartography, surveying, geostatistics, web mapping, programming, databases management and graphic design. Moreover, GIS also played an important role in integrating data from different sources to provide geographical information that proved to be critical for relief operations.

One of the applications of GIS is disease management. Many countries have used GIS and spatial evaluation to find out about the TB disease distribution and have shown a distinct geographical clustering of TB cases, suggesting the probability of ongoing transmission in that area. This can be proven by one of the previous studies from Malaysian Journal of Public Health Medicine 2018. It was about a geographical information system (GIS) application in tuberculosis spatial clustering 
studies. Geographical Information Systems (GIS) and spatial analyses have been utilized to depict the spatial distribution and clustering of different irresistible illnesses counting Tuberculosis (TB).

There are many implied, and advantages when sharing the human and environmental geospatial databases in public. The public can access the location that is affected with any kind of disease easily with GIS. Geographers utilize an extent of gear to ponder these subjects such as maps, statistics, and Geographic Information Systems (GIS). GIS may be a computer framework consisting of equipment and programs utilized to store, control, analyse, show, and show spatial and non-spatial information. GIS databases used in public health usually contain raster and vector file formats. The vector data uses points, lines and polygon to represent spatial features with clear spatial location and boundary. Spatial statistics can be used to analyze the lifestyle factors of a disease.

Spread of numerous illnesses inside a populace characterizes a spatial component. Spatial analysis tools such as GIS and spatial insights permit disease transmission experts to handle the spatial conveyance and to foresee the flare-ups of maladies more precisely (Chaput et al., 2002). Spatial investigation or spatial insights incorporate any of the formal strategies which ponder substances the utilization of their topological, geometric, or geographic properties. Spatial investigation comprises an assortment of procedures. The utilization of diverse expository methods and utilized in areas as differing as astronomy, with it investigating the situation of worlds within the universe, to chip manufacture designing, with it utilizing "place and route" algorithms to construct complicated wiring structures. In an additional restricted sense, spatial investigation is the approach connected to buildings at the human scale, most massively within the investigation of geographic information.

Spatial analysis stands up to numerous basic issues within the definition of its objects of consider, within the development of the explanatory operations to be utilized, within the computer frameworks for examination, within the restrictions and particularities of the investigations which are known, and within the introduction of analytic comes about. In human diseases and lifestyles, spatial cluster analysis plays a vital role in quantifying geographic variation patterns. Patterns and relationships in the data based on geography through spatial analysis can be detected. The map by Dr. John Snow of London, showing clusters of cholera cases in the 1854 Broad Street cholera outbreak. This was one of the first uses of mapbased spatial analysis. This shows that spatial analysis was used for the last century. Nowadays, spatial analysis is still used in any field, especially in human diseases and has been improving day by day.

People's opinion and participation in decision making also assisted GIS in creating a better mapping and spatial cognitive planning. Abdul Rasam et al., (2020b) addressed that local spatial knowledge (LSK) is developed by using local experts or community knowledge, as well as spatial cognition, to acquire data and create a crisis risk management decision-making plan. Using interactive web-based workshops to educate landscape simulations of conservation scenarios can also help to strengthen the role of expert knowledge within a community. In the context of health and epidemiology, a spatial knowledge-based method is desired to assess the risk factors and their relative risk weights in order to map the areas appropriate for disease transmission. Landscape simulation among the public on an issue can be enhanced by using public participatory GIS (PPGIS). The term PPGIS was established in two sessions of the PPGIS, which are the National Centre for Geographic Information and Analysis (NCGIA) as the next generation of GIS that would allow for technological breakthroughs in social and political settings while also, being more open to non-official voices (Giuffrida et al., 2019). The resulting definition of PPGIS centred on practical techniques including the public in GIS applications with the goal of boosting government policy transparency and influence.

\section{Materials and Methods}

Methodology is the important part applied in this study which covers the data collection, data creation, data processing and data analysis as shown in Figure 1.

\subsection{Study Area}

The study area was conducted at Section 7 Shah Alam. Shah Alam is located in Selangor, Malaysia. Shah Alam is one of the areas that had a higher population than other areas in Selangor. In addition, Shah Alam is one of the towns which have been rapidly developing. This may influence the lifestyle effect on TB disease. Besides applying the PPGIS approach, this study also applied spatial vector analysis and geostatistical analysis for exploring the spatial interaction between lifestyles and TB cases (Figure 2). 


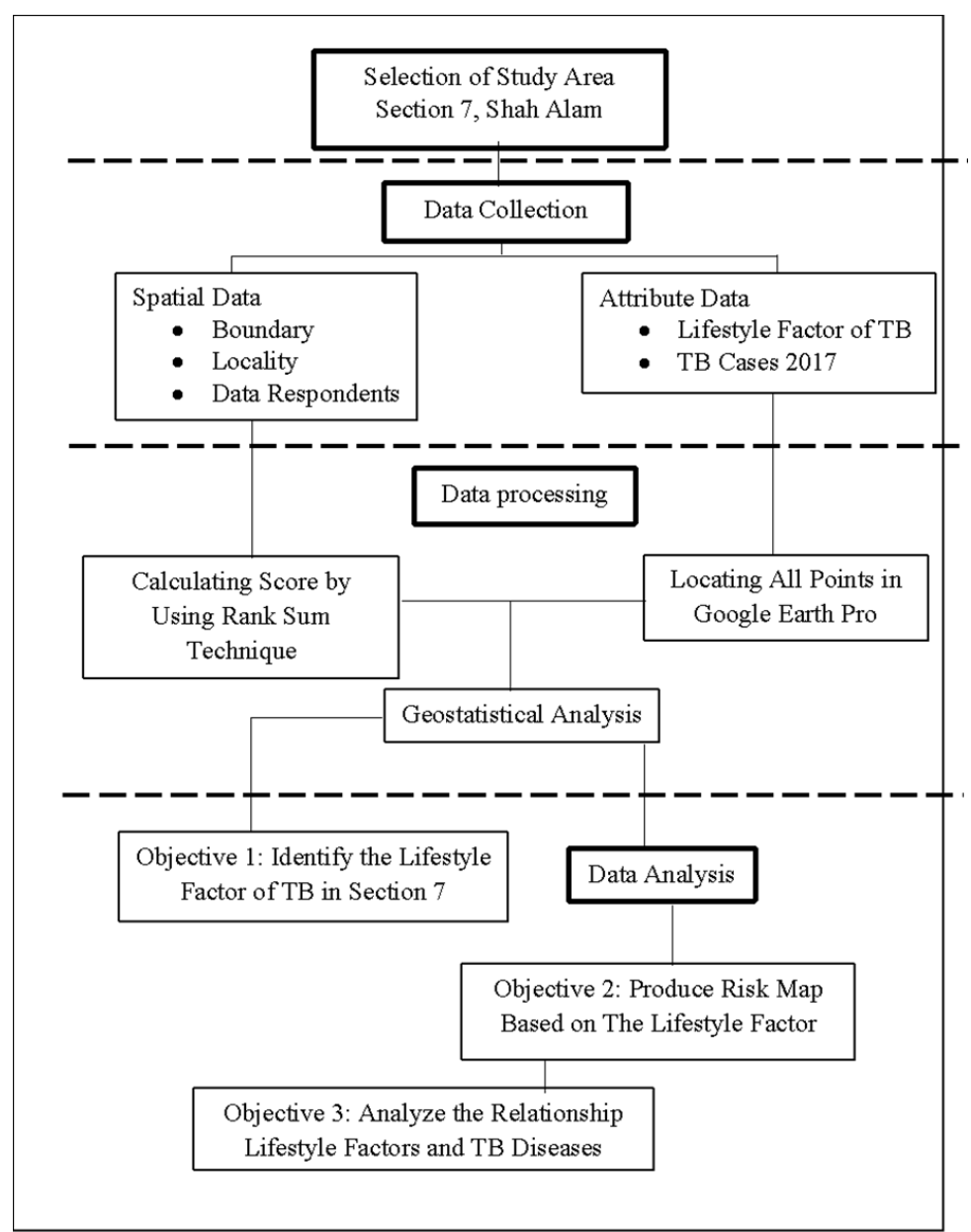

Figure 1: Research methodology

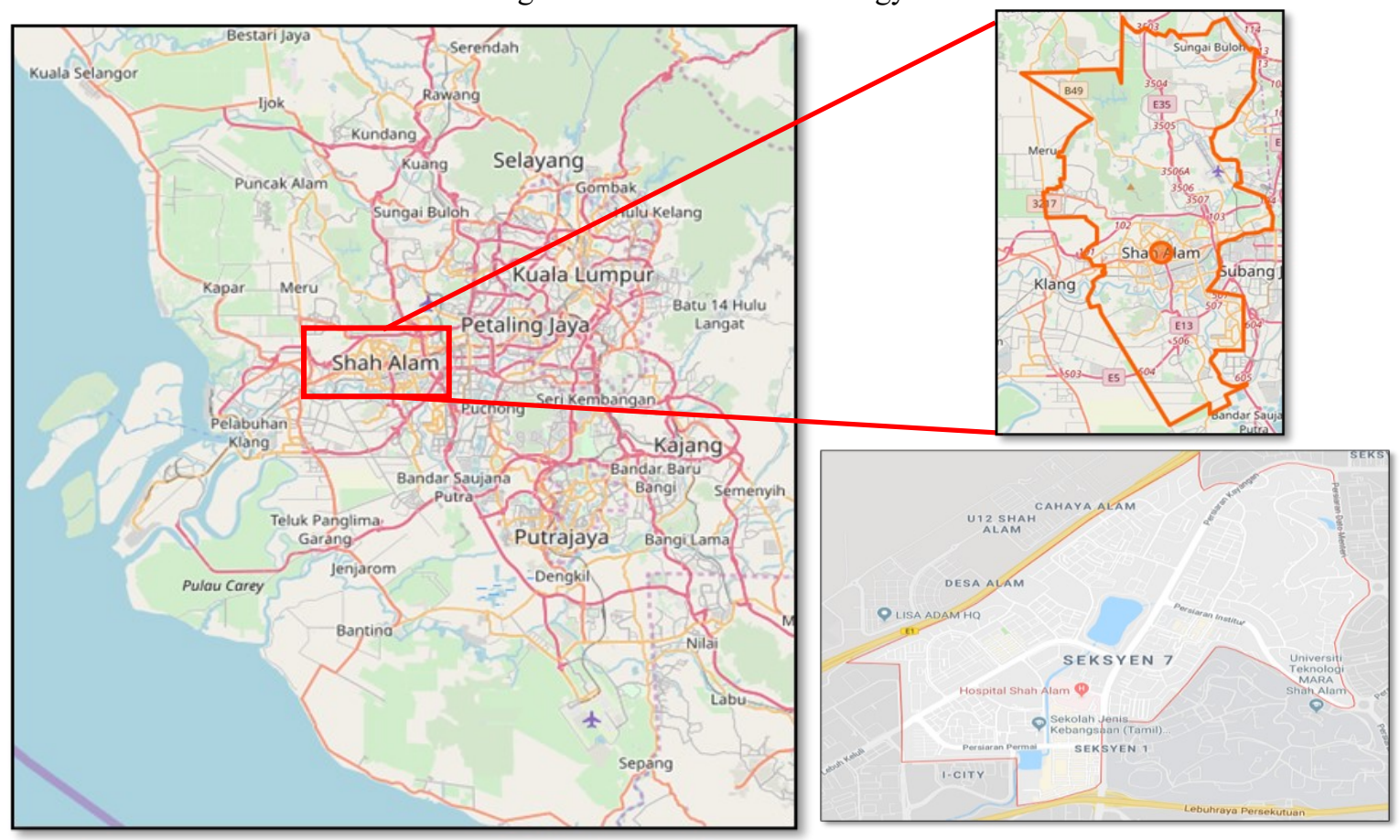

Figure 2: Location of Section 7, Shah Alam in Selangor (Sources: www.openstreetmap.org) 


\subsection{Data collection and Database}

3.2.1 Research questionnaire for acquiring nonspatial data of the risk factors

The questionnaires were disseminated to the respondents (Table 1). Additional information and some comments were recorded as well. For example, the address should be in details including the house number and section. The other non-spatial data are nine selected lifestyle factors. In addition, based on the research questionnaire, there are a few social-demographic questions. Socio-demographic is categorized as non-spatial data. The sociodemographic is divided into a group such as gender, age, race, education, household income and also occupation. The final total data collected was 100 respondents. Four opinions of the local public health in the state were also obtained for risk factor ranking and selection using rank sum techniques.

\subsubsection{Spatial data}

Collecting data on spatial data from the research questionnaire related the lifestyle factors of TB diseases was also conducted. The spatial data in this research include the address of the respondent that can get the location of $\mathrm{x}$ and $\mathrm{y}$ on the risk map. The base map of Selangor state was obtained from the Department Surveying and Mapping Malaysia (JUPEM). The thematic map was digitized from the base map of Selangor from the scanning image format such as .JPEG, .TIFF an.IMG. Other than that, the base map can also be obtained from ArcGIS Online. The information contains the boundary of Shah Alam area and spatial data were carried out and performed using GIS software. Figure 3 shows the main residential localities of the area around section 7. The localities are identified and referred to through Google Earth Pro, Google Map, Google Street and also site visit. After identifying the locality of the Section 7 Shah Alam, the data of the respondent are pinned in the Google Earth Pro. It is to show the distribution of the data. Figure 4 shows the distribution of data collected from online and offline method collection, representing the accepted respondents in the study area.

Table 1: Questionnaire on the lifestyle factors

\begin{tabular}{|c|c|c|c|c|}
\hline Never & Rarely & Sometimes & Often & Always \\
\hline 1 & 2 & 3 & 5
\end{tabular}

\begin{tabular}{|ll|l|l|l|l|l|}
\hline \multicolumn{2}{|l|}{ Lifestyle factors } & \multicolumn{5}{|c|}{ Scale } \\
\hline 1$)$ & Do you an active smoker? & 1 & 2 & 3 & 4 & 5 \\
\hline 2$)$ & Do you drink alcohol? & 1 & 2 & 3 & 4 & 5 \\
\hline 3$)$ & Do you have problems in physical exercise involvement? & 1 & 2 & 3 & 4 & 5 \\
\hline 4$)$ & Do you have problems in taking take a good diet in your meals? & 1 & 2 & 3 & 4 & 5 \\
\hline 5$)$ & Do you have problems in having an ideal weight? & 1 & 2 & 3 & 4 & 5 \\
\hline 6$)$ & Do you have a socioeconomic status problem? & 1 & 2 & 3 & 4 & 5 \\
\hline 7$)$ & Does your living crowded condition? & 1 & 2 & 3 & 4 & 5 \\
\hline 8$)$ & Have you been infected with HIV infection? & 1 & 2 & 3 & 4 & 5 \\
\hline 9$)$ & Do you have a family history of diabetes? & 1 & 2 & 3 & 4 & 5 \\
\hline
\end{tabular}




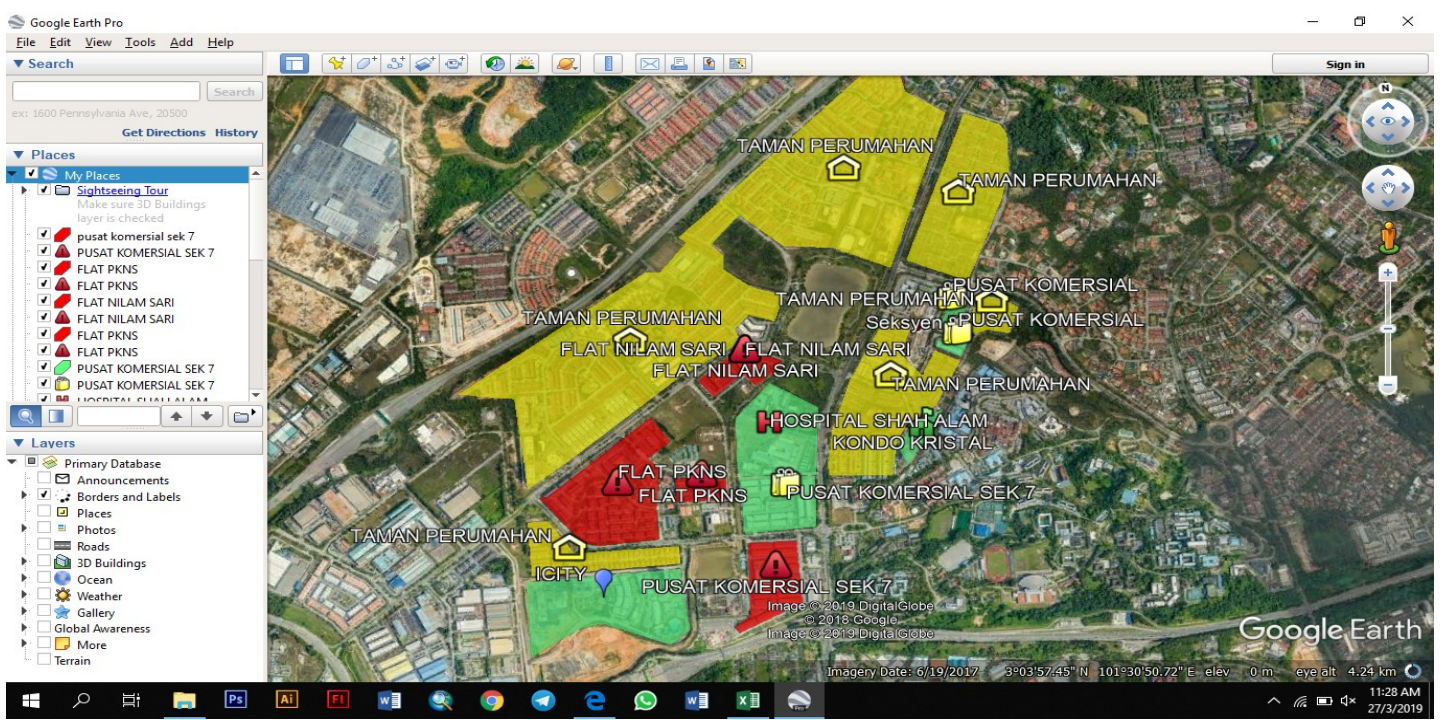

Figure 3: The Identification of the locality of risk area in Section 7 Google Earth Pro

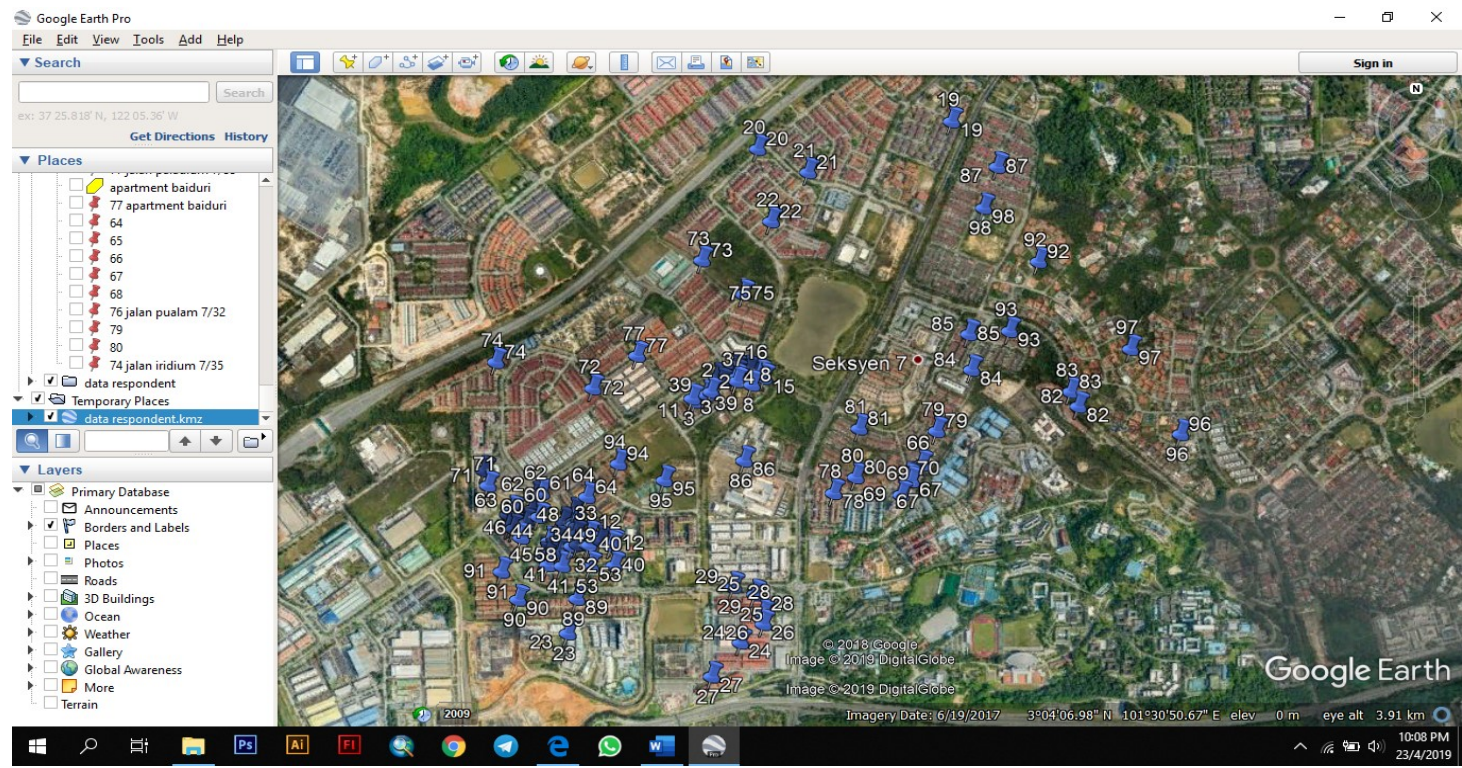

Figure 4: The Data of respondents in Section 7 pinned in Google Earth Pro

Table 2: Scoring TB-lifestyle factor based on the two expert opinions from the health department

\begin{tabular}{ccc}
\hline $\begin{array}{c}\text { Respondent } \mathbf{l} \\
\text { Selected Lifestyle Risk Factors }\end{array}$ & Scale & Score \\
& & \\
\hline Smoking & 1 & \\
Alcoholism & 1 & \\
Physical exercise & 3 & \\
Diet/nutrient & 3 & \\
Weight & 4 & 2.33 \\
Socioeconomic status & 3 & \\
crowded condition & 4 & \\
HIV/Diseases infection & 1 & \\
History of diabetes & 1 & \\
TOTAL & 21 & \\
\end{tabular}




\subsubsection{Data Processing and Data Analysis}

The risk of lifestyle factors was calculated by expert opinion and public opinion (respondents). The risk level of the expert opinion was computed using rank sum technique. For example, respondent 1 has scored 2.33 the lifestyle of TB risk as shown in Table 2. While for public opinion used a 5 likert scale of risk (0.01to1=very low [dark green], 1.01 to2=low [light green], 2.01 to3medium [yellow], 3.01 to4 [orange], 4.01to5=very high [red]) based on the questionnaire (Table 1) and ArcGIS mapping (IDW-geostatistical approach). The other software also used is a Microsoft Excel to arrange the data and create a graph of the risk level of the factors.

Inverse Distance Weighting (IDW) is a speedy deterministic method used for visualizing the risk level of the factors only. The final step was to produce the risk map of TB diseases. Such maps would make it conceivable to arrange control measures in high-risk ranges and enormously increment the fetched effectiveness of these control programs. These spatial data methods can be compelling apparatuses in TB checking and observation contributing to fill the crevices within the current understanding of illness dispersion. GIS and inaccessible detecting methods were utilized to outline the spatial conveyance of TB illnesses and potential risk areas. The risk map shows the risk of TB rate in Shah Alam created with the ArcMap.

\section{Results and Discussion}

This part details the result of the study. It also focuses on analysis and implications of the lifestyle factors study on the TB among the society in Shah Alam, a risk factor map of lifestyle effect on airborne disease in Shah Alam and the other objective is to determine the qualitative relationship between lifestyle factors and TB occurrences.

\subsection{The Lifestyle Risk Factors of TB Diseases}

The lifestyle risk factors are used to identify the percentage that influences TB. Nine common lifestyle factors are selected since there is no a specific standard guideline used for global preferences. Based on the public-questionnaire conducted (Figure 5), the overall average risk level of lifestyle factors is 2.0-2.50 (a low-medium risk), or more specifically is 2.08 (a medium risk). This public opinion result was consistent with result found from the expert opinion (Table 2) is 2.33 (a medium risk), also in a medium risk. Based on the level risk of the respective risk factors, status of obesity and diet of the community recorded a quite high risk scenario, followed by the condition of living, physical exercise, socio-economic, and diabetes that stated in a medium risk. Status of smoking, alcoholism and HIV are selected as a low risk.

Both opinions have suggested that the local communities need to emphasize food and body care because these two factors have the potential to cause TB disease due to the reduction of the immune system in the body. Weyh et al., (2020) suggested that lifestyle factors, such as physical activity and diet habits, significantly affect the process of immunosenescence and inflammaging. Accordingly, regular exercise training and specific nutrition strategies support successful immune aging and decrease the risk of maladaptive immune aging. If we are healthy, we most likely have a robust immune system and our bodies can readily fight off diseases caused by bacteria or viruses, such as TB. In addition, a low immune system will be worse if a person has a low income (Lopez et al., 2008), has other illnesses (Jeon and Murray, 2008, Leung et al., 2007 and Lin et al., 2018) and lives in a crowded area (Baker et al., 2008, Andrejs et al., 2016 and Yen et al., 2017). This is because each of these factors is interrelated and becomes more contagious if these factors are combined together.

The next section is a detail on the condition or status of each of the factors of the study area. First, referring to smokers among Section 7 residents, the highest percentage is $85 \%$ who had never smoked. $6.2 \%$ are rarely smokers and $5.3 \%$ are sometimes smokers. The often smokers show 3.5\% over the total respondent's result. The Section residents are not an active smoker. The overall smoking level is only 1.57 (low). In terms of alcoholism, $92.9 \%$ of the Section 7 residents are not alcohol drinkers. Most of the respondents who are alcohol drinkers are non-Islamic communities. The percentage of alcohol drinkers at the scale 2 is $2.7 \%$ only; meanwhile the $3.5 \%$ is quite often drinker. The alcohol level is 1.22 (low). While for physical exercise, $61.1 \%$ are active in sport. Based on the site visit around Section 7, they usually exercise such as playing badminton, running and playing football. The percentage of rarely taking part in physical exercise is $22.1 \%$ and often is $11.5 \%$. Meanwhile the other is at low percentages. The physical exercise level is 2.92 (medium). 


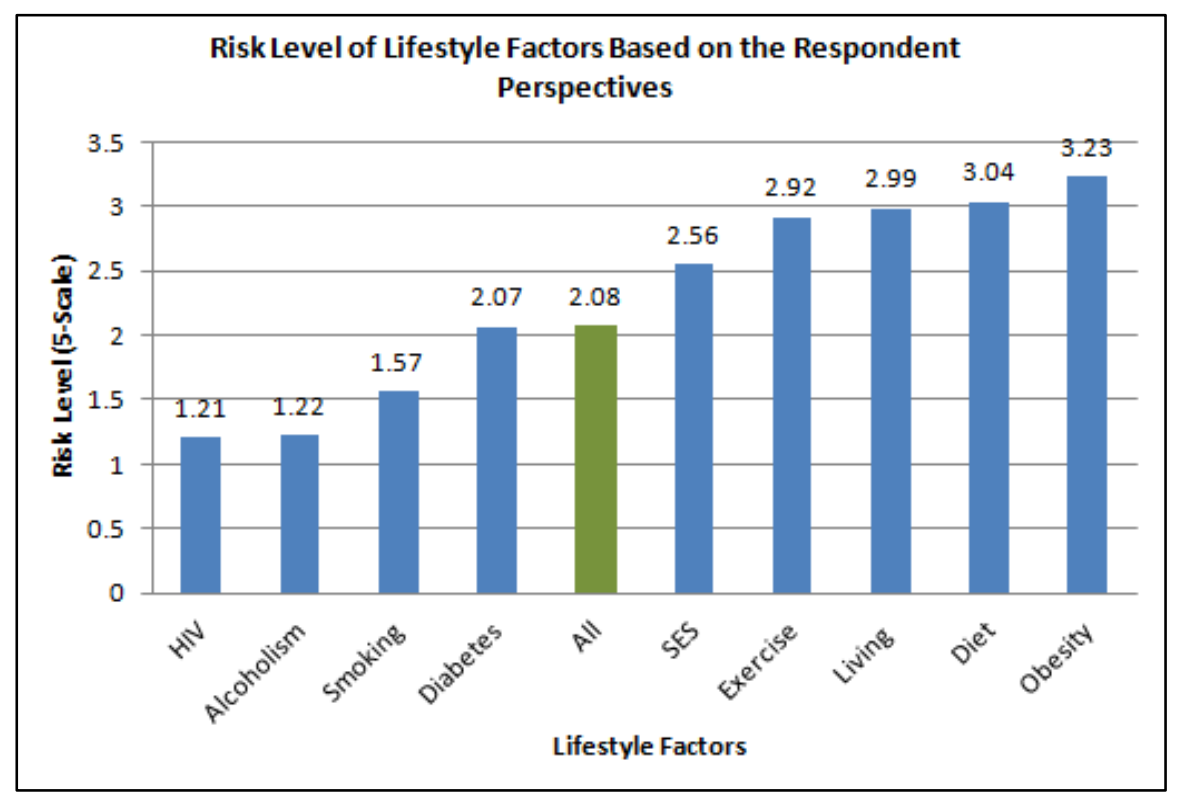

Figure 5: Risk level of lifestyle factors according to the respondent feedbacks

Based on the respondents' result on nutritional status has shown that $62.8 \%$ are taking a good diet in their meals. The second highest percentage is $19.5 \%$ which is rarely scale. One of the reasons that they cannot manage to have a good diet is because they are too busy with their work. The percentage who always take a good diet is $4.4 \%$ only. The nutritional status is medium. Regarding the obesity problem among Shah Alam residents. The highest percentage is $26.5 \%$; the second highest is $25.7 \%$ and followed by $24.8 \% .7 .1 \%$ of them have never faced the obesity problem.

The obesity level is medium. Everyone in the residents has socioeconomic problems. It can be in many ways. One of the way is a financial problem. Only $2.7 \%$ always have a socioeconomic problem. $48.7 \%$ are at the average level. In the previous studies, TB rates were highest in the quartile with low SES (Andrejs et al., 2016, Cramm et al 2011, Kashyap et al., 2016, Lopez De Fede et al., 2008, Muniyandi et al., 2011, Olson et al., 2012, Yen et al., 2017 and Abdul Rasam et al., 2016) both and foreign-born populations.

The socioeconomic status is medium. In addition, Living condition affected the TB among respondents. $29.2 \%$ of the Section residents are staying in medium scale of the crowded area. But $8 \%$ of them are staying in high-density of population in Section 7. The living condition level is medium. Most of the residents have never been influenced by HIV. There are $95.6 \%$ of them. On the other scale is in low percentage. Based on the WHO, TB deaths among HIV-negative people per 100000 population per year) is falling at about $3 \%$ per year, and the overall reduction in the period 2000-2017 was $42 \%$. The HIV level is low. Lastly, the percentage of history of diabetes level among respondents is medium. $42.5 \%$ have never had a family history of diabetes. At the scale 2 and 3 are in the medium percentage. $7.1 \%$ have been influenced by diabetes.

\subsection{Mapping the Correlation between Lifestyle Factors and TB Diseases}

Figure 6 shows the risk map of lifestyle factors related to historical TB occurrences at Section 7. Although the distribution of the disease is random pattern, the cases are stilled clustered in certain localities, for example, in Flat PKNS, Flat Nilam, Jalan Platinum. In general, the concentration of TB occurrences is not directly correlated with the status of lifestyle factors due to the minimal significant correlation between these variables using geospatial-epidemic analysis.

But, in -depth studies need to be conducted for some of the lifestyles factors that have the potential to affect the local TB epidemics. Most of the cases occurred in a medium risk (1-3 scale). This reveals that TB can attack any person of having lifestyle risk, but the more risk will occur on certain risk factors such as a person suffering from two noncommunicable diseases at the same time (Figure 7). For example, the risk map of the physical exercise status shows Shah Alam has medium to high risk level. The medium risk level is within3.0 to 3.5. Meanwhile, high risk level within 4 to 5 . The estimated risk level is medium-high. 


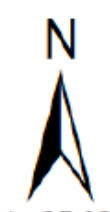

$1: 25000$

Risk Level

$0-0.5$

$0.5-1$

$1-1.5$

- $1.5-2$

$2-2.5$

$2.5-3$

- $3-3.5$

$3.5-3.68$

- TB Cases
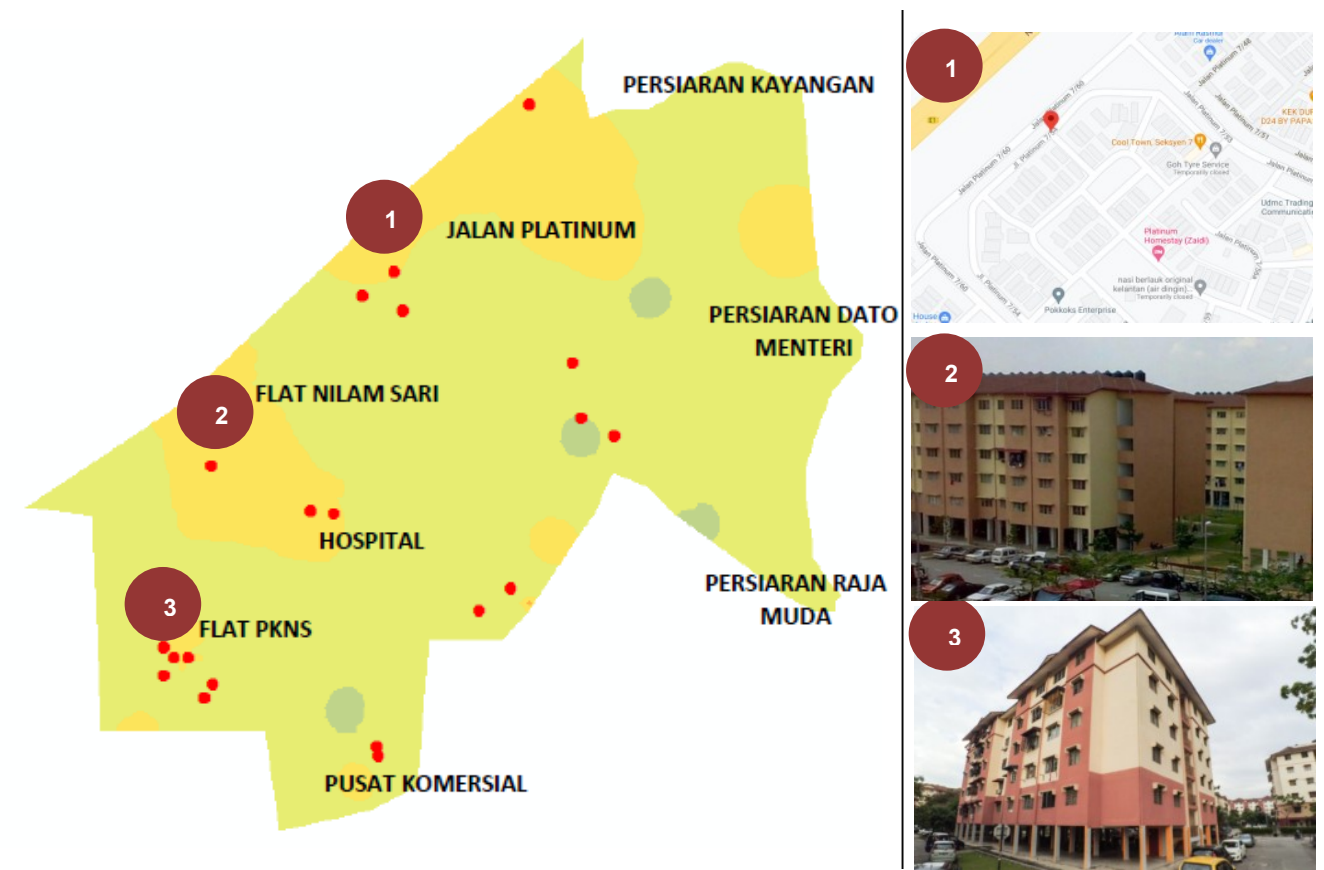

Figure 6: The risk map of lifestyle factors

Distribution of Risk Level of Lifestyle \& TB
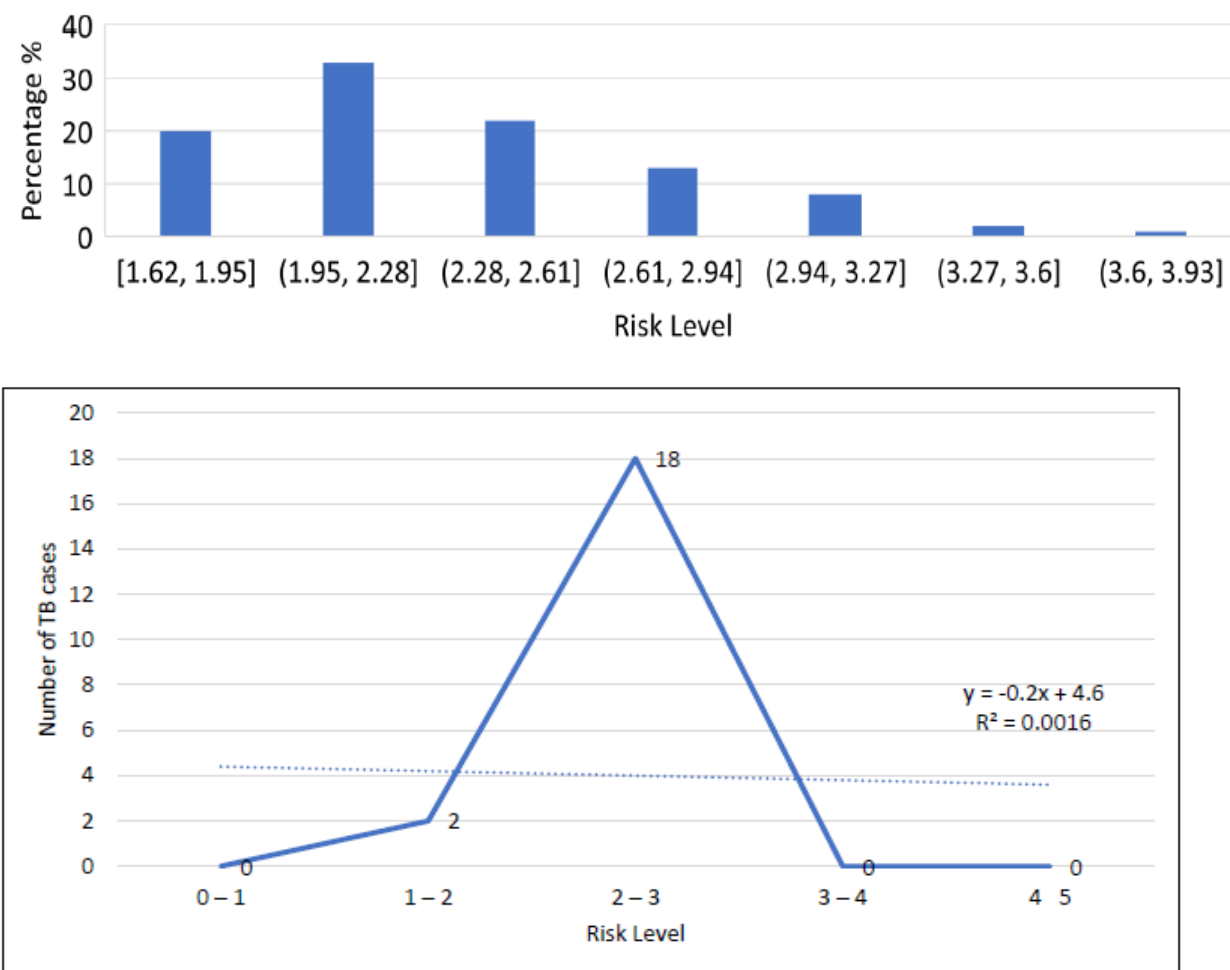

Figure 7: The risk level of lifestyle factors

For the risk map of nutritional status, it shows that shows Shah Alam has low risk level. The risk level is in between 2.0 to 4.0. The area in between Hospital Shah Alam and Flat PKNS is 0 to 0.5 risk level. The estimated risk level is medium-high.
However, the impact of the factors (physical exercise status and nutritional status) will be effective to attack a person to get TB infection if they are combined together. Therefore, Ngai. (2002) emphasized the best weapon is our own defence 
system by paying attention to personal hygiene, adopting a healthy lifestyle and eating a wellbalanced diet, getting adequate rest and exercise, not smoking or drinking alcohol, keeping the environment clean and avoiding poorly ventilated areas.

\section{Conclusion}

In conclusion, in this exploratory context, there is a minimal correlation between the nine selected lifestyle factors and TB disease. However, certain risk factors have potential to influence to others due to each of these factors is also interrelated and becomes more contagious if these factors are combined together. For example the poor living condition, weak physical exercise and inadequate nutritional routine seem have related to the disease occurrences. Both diet and physical activity also play a critical role in maintaining a healthy body weight, hence inadequate nutrition or problematic weight will associate with increased susceptibility to the infection. The findings suggested that public health actions are needed to promote education on TB patients, about the significance of nutritional support and other aspects related to the risk factors. It is also necessary to develop a detailed guideline for these factors, such as the guideline in physical activity in with a precise onset of the rehabilitation programs, adapted and individualized exercise types, as well as other measures for assessing the outcomes. In terms of approach, by using a people opinion and a GIS, the risk area of the TB could be produced and analysed properly. Next study will be applying advanced geocomputation techniques and comprehensive data collection for quantifying the relationship and modelling development.

\section{Acknowledgements}

The authors gratefully acknowledge the assistance of the Ministry of Higher Education (MOHE) and Universiti Teknologi MARA Selangor for providing Fundamental Research Grant Scheme (FRGS) 600IRMI/FRGS 5/3 (093/2019). The research is registered in the National Medical Research Register, Malaysia (ID: NMR R -15-2499-24207).

\section{References}

Abdul Jalil, I. and Abdul Rasam, A. R., 2021, Social Network Analysis of Spatial Human Mobility Behaviour in Infectious Disease Interaction: An Exploratory Evidence Of Tuberculosis In Malaysia, Int. Arch. Photogramm. Remote Sens. Spatial Inf. Sci. XLIII-B4-2021, 55-61, https://doi.org/10.5194/isprs-archives-XLIII-B42021-55-2021.
Abdul Rasam, A. R., Shariff, N. M. and Dony, J. F., 2016, Identifying High-Risk Populations of Tuberculosis Using Environmental Factors and GIS Based Multi-Criteria Decision Making Method. The International Archives of the Photogrammetry, Remote Sensing and Spatial Information Science, Vol. XLII-4/W1, 9-13. https://doi.org/10.5194/isprs-archives-XLII-4W1-9-2016, 2016

Abdul Rasam, A. R., Shariff, N. M., Dony, J. F. and Punitha, M., 2017, Mapping Risk Areas of Tuberculosis Using Knownledge-Driven GIS Model in Shah Alam, Malaysia. Pertanika $J$. Soc. Sci \& Hum, Vol. 25(S), 135-144.

Abdul Rasam, A. R., Mohd Shariff, N. and Dony, J., 2020a, The Invention of Geospatial Decision Support System for Malaysian Tuberculosis Surveillance Data Management. EnvironmentBehaviour Proceedings Journal, Vol. 5(SI3), 269-274. https://doi.org/10.21834/ebpj.v5iSI3.2564

Abdul Rasam, A. R., Mohd Shariff, N., Dony, J. and Ling, O. H. L., 2020b. Local Spatial Knowledge for Eliciting Risk Factors and Disease Mapping of Tuberculosis Epidemics. EnvironmentBehaviour Proceedings Journal, Vol. 5(SI2), 4551. https://doi.org/10.21834/ebpj.v5iSI2.2522

Andrejs, I., Ieva, S. K. and Ludmila, V., 2016, The Impact of Socioeconomic Factors on Tuberculosis Prevalence in Latvia. Universal Journal of Public Health, Vol. 4(5), 230-238.

Baker, M., Das, D., Venugopal, K. and HowdenChapman, P., 2008, Tuberculosis associated with household crowding in a developed country. Journal of Epidemiology and Community Health (1979-), Vol. 62(8), 715-721. [Available Online At http://www.jstor.org/stable/40665887].

Booth, F. W., Roberts, C. K. and Laye, M. J., 2012, Lack of Exercise is a Major Cause of Chronic Diseases. Comprehensive Physiology, Vol. 2(2), 1143-1211. https://doi.org/10.1002/cphy.c110025 .

Brunet, L., Pai, M., Davids, V., Ling, D., Paradis, G. and Lenders, L., 2011, High Prevalence of Smoking among Patients with Suspected Tuberculosis in South Africa. European Respiratory Journals, 139-146.

Centers for Disease Control and Prevention USA [CDC], 2015, Division of Tuberculosis Elimination, National Center for HIV, Viral Hepatitis, STD, and TB Prevention, Centers for Disease Control and Prevention. TB and HIV Coinfection. https://www.cdc.gov/tb/topic/basics/tbhivcoinfection.htm. 
Centers for Disease Control and Prevention USA [CDC], 2011, Tuberculosis (TB). https://www.cdc.gov/tb/publications/factsheets/g eneral/tb.htm

Chang, K. C. and Leung, C. C., 2008, Impact of Lifestyle on Tuberculosis. Respirology, Vol.13, 65-72.

Chang, K., 2019, Introduction to Geographic Information System. Nineth Edition. New York: McGraw-Hill.

Chaput, E. K., Meek, J. I. and Heimer, R., 2002, Spatial Analysis of Human Granulocytic Ehrlichiosis Near Lyme, Connecticut. Emerging Infectious Diseases, Vol. 8(9), 943-948. https://doi.org/10.3201/eid0809.020103.

Cramm, J. M., Koolman, X., Moller, V. and Nieboer, A. P., 2011, Socio-economic Status and Self-Reported Tuberculosis: A Multilevel Analysis in a Low-Income Township in the Eastern Cape, South Africa. Journal of Public Health in Africa, 143-146. DOI:10.4081/jphia.2011.e34.

Cromley, E. K., 2003, GIS and Diseases. Annu. Rev. Public Health, 7-24.

Davis, G. S., Sevdalis, N. and Drumright, L. N., 2018, Spatial and Temporal Analyses to Investigate Infectious Diseases Transmission within Healthcare Settings. Malaysian Journal of Public Health Medicine, Vol. 86(4), 227-243.

European Respirotry Society [ERS], 2021, Tobacco smoking. https://www.erswhitebook.org/chapters/tobacco-smoking/.

Farhud, D. D., 2015, Impact of Lifestyle on Health. Iran J Public Health, 1442-1444.

Giuffrida. N., Le Pira, M., Inturri, G., Ignaccolo. and M., 2019, Mapping with Stakeholders: An Overview of Public Participatory GIS and VGI in Transport Decision-Making. ISPRS International Journal of Geo-Information, Vol. 8(4), 198. https://doi.org/10.3390/ijgi8040198.

Ibrahim, H. M., Badia, M. N. and Namir, G. A., 2011, Association between Lifestyle Factors and Pulmonary Tuberculosis in Erbil. Journal of Medical Sciences, Vol. 15(3), 6-11.

JAMA and Archives Journals, 2007, Smoking May Be A Risk Factor for Tuberculosis. ScienceDaily. Retrieved October 10, 2021. [Available online at www.sciencedaily.com/releases/2007/02/070227105634.htm].

Jeon, C. Y. and Murray, M. B., 2008, Diabetes Mellitus Increases the Risk of Active Tuberculosis: A Systematic Review of 13 Observational Studies. PLoS Med, Vol. 5(7), 111
Kashyap, R. S., Nayak, A. R. and Husain, A. A., 2016, Impact of Socioeconomic Status and Living Condition on Latent Tuberculosis Diagnosis among the Tribal Population of Melghat: A Cohort Study. Lung India, 372-380.

Leung, C. C., Lam, T. H. and Chan, W. M., 2007, Lower Risk of Tuberculosis in Obesity. Arch Intern Med, 1297-1304.

Lienhardt, C., Fielding, K., Sillah, J. and Tunkara, A., 2003, Risk Factors for Tuberculosis Infection in Sub-Saharan Africa. American Journal of Respiratory and Critical Care Medicine, Vol. 168(4), 448-455.

Lin, C. Y., Chen, T. C., Lu, P. L., Lai, C. C. and Yang, Y. H., 2013, Effects of Gender and Age on Development of Concurrent Extrapulmonary Tuberculosis in Based Cohorts. Clinical Infectious Diseases, Vol. 66(5), 699-705.

Lonnroth, K., Williams, B., Cegielski, P. and Dye, C., 2010, A Consistent Patients with Pulmonary Tuberculosis: A Population Based Study. PLoS ONE, Vol. 8(5), e63936. doi:10.1371/journal.pone.0063936.

Lin, H. H., Wu, C. Y., Wang, C. H., Fu, H., Lonnroth, K., Chang, Y. C. and Huang, Y. T., 2018, Association of Obesity, Diabetes, and Risk of Tuberculosis: Two Population -Linear Relationship between Tuberculosis Incidence and Body Mass Index. International Journal of Epidemiology, Vol. 39(1), 149-155.

Lopez, D.F, A., Stewart, J. E., Harris, M. J. and Mayfi eld-Smith, K., 2008, Tuberculosis in Socio-Economically Deprived Neighborhoods: Missed Opportunities for Prevention. The International Journal of Tuberculosis and Lung Disease, Vol. 12(12), 1425-1430.

Mohammed, T., Amare, D., Fasil, T., Sahilu, A., Luc, D. and Robert, C., 2011, Risk Factors of Active Tuberculosis in People Living with HIV/AIDS in Southwest Ethiopia: A Case Control Study. Ethiop J Health Sci, Vol. 21(2),131-139.

Muniyandi, M., Ramachandran, R., Balasubramanian, R. and Narayanan, P. R., 2006, Socio-economic Dimensions of Tuberculosis Control: Review of Studies over Two Decades from Tuberculosis Research Center. J. Commun. Dis, Vol. 38 (3), 204-215.

Narasimhan, P., James, W., Chandini, R. M. and Dilip, M., 2013, Risk Factors for Tuberculosis. Pulmonary Medicine, 1-11, DOI:10.1155/2013/828939. 
Ngai, A., 2002, Healthy Lifestyle the Best Way to Prevent Tuberculosis. South China Morning Post. [Available online at https://www.scmp.com/article/389649/healthy-lifestyle-bestway-prevent-tuberculosis].

Olson, N. A., Davidow, A. L., Winston, C. A., Chen, M. P., Gazmararian, J. A. and Katz, D. J., 2012, A National Study of Socioeconomic Status and Tuberculosis Rates by Country of Birth, United States. BMC Public Health, Vol. 12(1), 1-7.

Rajab, N. A., Hashim, N., and Rasam, A. R. A., 2020, Spatial Mapping and Analysis of Tuberculosis Cases in Kuala Lumpur, Malaysia. 2020 IEEE 10th International Conference on System Engineering and Technology (ICSET), 38-43. doi: 10.1109/ICSET51301.2020.9265385

Rasam, A.R.A., Mohd Shariff, N., F Dony, J., and Misni, A., 2018, Socio-Environmental Factors and Tuberculosis: an Exploratory Spatial Analysis in Peninsular Malaysia. International Journal of Engineering \& Technology, Vol. 7(3.11), 187-192. doi:http://dx.doi.org/10.14419/ijet.v7i3.11.15958

Rasam, A. R. A., Shariff, N. M., Dony, J. F. and Omar, D., 2019, Sociospatial Risk Assessment of Human-Environment-Tuberculosis Interactions in Rural-Urban Settings. Malaysian Construction Research Journal, Vol. 8(3), 177187.

Rosli, N. M., Shah, S. A. and Mohmood, M. I., 2018, Geographical Information System (GIS) Application in Tuberculosis Spatial Clustering Studies: A Systematic Review. Malaysian Journal of Public Health Medicine, 70-80.
Suhadev, M., Thomas, B.E., Raja Sakthivel, M., Murugesan, P., Chandrasekaran, V., Charles, N., Durga, R., Auxilia, M., Mathew, T. A. and Wares, F., 2011, Alcohol Use Disorders (AUD) among Tuberculosis Patients: A Study from Chennai, South India. PLoS One. Vol. 6(5), e19485. doi: 10.1371/journal.pone.0019485.

Tocque, K., Bellis, M. A., Beeching, N. J., Syed, Q., Remmington, T. and Davies, P. D. O., 2001, A Case Control Study of Lifestyle Factors Associated with Tuberculosis in Liverpool, North-west England. European Respiratory Journals, 959-964.

Weyh, C., Krüger, K. and Strasser, B., 2020, Physical Activity and Diet Shape the Immune System during Aging. Nutrients, Vol. 12(3), 622. [Available online at https://doi.org/10.3390/nu12030622].

Yen, Y. F., Hu, H. Y., Lee, Y. L., Ku, P. W., Lin, I. F., Chu, D. and Lai, Y. J., 2017, Obesity/Overweight Reduces the Risk of Active Tuberculosis: A Nationwide Population-Based Cohort Study in Taiwan. International Journal of Obesity, 971-975. 